\section{Versatile dental cement}

When using dental cement you want a solution that can be used in a variety of applications.

RelyX Unicem self-adhesive resin cement from 3M Oral Care offers true versatility.

It is suitable for use on bridges, crowns, screws, inlays and onlays. One clinical study even found the product to be ideal for the cementation of root canal posts as it showed no signs of debonding after a five-year period.

Furthermore, RelyX Unicem has been specifically designed to offer great aesthetics due to its natural looking tooth-like fluorescence, meaning it is suitable for creating aesthetic indications.

For more information, call 0800626578 or visit www.3M.co.uk/Dental.

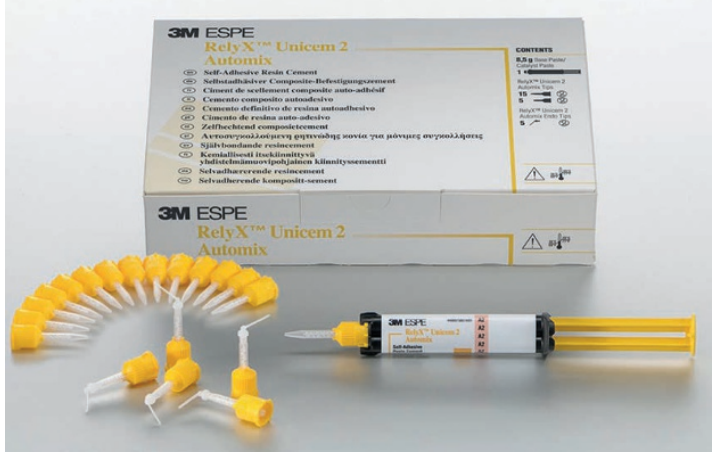

\section{A soft-tissue-friendly implant solution}

Nobel Biocare has recently added a new string to its bow with the introduction of NobelPearl implants. A soft-tissuefriendly implant solution, NobelPearl is a $100 \%$ metal-free, two piece ceramic implant with an internal cement-free locking mechanism that provides a unique alternative to titanium.

Geared towards aesthetic excellence, these implants help to support the natural soft tissue appearance and are especially useful for cases where patients may have a thin gingival biotype.

Furthermore, as the implants are made from zirconia they have been designed to encourage excellent soft tissue attachment and minimise inflammatory response, encouraging predictable results.

Combine these benefits with a low plaque affinity and natural looking aesthetics and you can see why Nobel Biocare's new solution is a treasure well worth discovering.

For more information, contact Nobel Biocare on 0208756 3300, or visit www.nobelbiocare.com/pearl.

\section{Complete prophylaxis solution}

Regular scaling and root planing is essential to minimising the development of periodontal disease. For optimal results, W\&H offers a complete range of high-performance solutions for prophylaxis.

The Proxeo air scaler with integrated spray and two large optic outlets ensures the gentle yet effective removal of plaque and calculus whilst cleaning the root surfaces. There are a number of tips available to ensure that whatever you're faced with, you can achieve quality results every time.

$\mathrm{W} \& \mathrm{H}$ also provides the Tigon+ ultrasonic treatment unit complete with a piezo scaler handpiece with LED ring. Using temperature-controlled fluid, the Tigon+ prevents irritation, even in patients with sensitive teeth.

With the additional option of the Proxeo contra-angle handpiece suitable for cleaning, polishing and fluoridation, dentists can safely and efficiently remove calculus to an extremely high standard, helping to reduce the risk of periodontal disease and boost patients' oral health.

To find out more visit www.wh.com/en_uk, call 01727874990 oremail office.uk@wh.com.

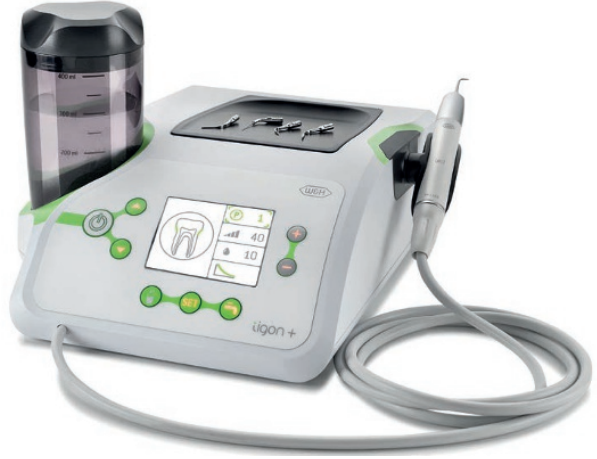

\section{One for all}

A simpler way to streamline restorative implant procedures, the On1 concept from Nobel Biocare offers dentists surgical flexibility like never before. Developed with making restorations more straightforward in mind, the On1 concept moves the restorative platform from bone level to tissue level. This preserves soft tissue attachment as the base remains in place throughout the lifetime of the restoration. It also means that impression taking and the attachment of restorative components becomes easier as the connection is readily accessible.

Designed with a unique prosthetic connection, the On1 concept can be paired with any of Nobel Biocare's conical implant systems, including NobelActive, NobelParallel, and NobelReplace. Due to this it has a wide variety of applications and can provide trusted levels of primary stability to numerous patient cases.

A proven solution that offers flexibility, strength and a simple workflow, the On1 concept from Nobel Biocare can truly revolutionise the way you provide restorations.

For more information, contact Nobel Biocare on 0208756 3300, or visit www.nobelbiocare.com/on1. 